\title{
PD1/PDL-1 Inhibitors: Emerging Hope for Bladder Cancer
}

\author{
Samina Qamar ${ }^{1, ~ *, ~ U z a i r ~ B a s h i r ~ C h a u d h a r y ~}{ }^{2}$ \\ ${ }^{1}$ Division of Geriatrics, Gerontology \& Palliative medicine, University of Texas Health Science Center, San Antonio, USA \\ ${ }^{2}$ Division of Hematology \& Oncology, University of California San Francisco, Fresno, USA
}

\section{Email address:}

saminaqamar@ymail.com (S. Qamar)

${ }^{*}$ Corresponding author

\section{To cite this article:}

Samina Qamar, Uzair Bashir Chaudhary. PD1/PDL-1 Inhibitors: Emerging Hope for Bladder Cancer. Journal of Cancer Treatment and Research. Vol. 5, No. 5, 2017, pp. 71-80. doi: 10.11648/j.jctr.20170505.11

Received: July 18, 2017; Accepted: August 11, 2017; Published: September 4, 2017

\begin{abstract}
Advanced bladder cancer (BC) has a poor prognosis with historically limited therapeutic options. Immunotherapy has emerged as a viable option for patients with advanced bladder cancer in the second line setting. Immune check point inhibitors have shown promising results in management of this disease. In this review we will discuss the recently evolving role of anti PD-1/PD-L1 inhibitors (programmed cell death protein-1 and programmed death ligand-1) for managing this disease.
\end{abstract}

Keywords: Cancer, Bladder, Immunotherapy, PD-L1, PD-1

\section{Introduction}

Bladder cancer (BC) is the second most commonly diagnosed genitourinary malignancy after prostate cancer. In the United Stated estimated numbers of new cases were 76,960 with approximately 16,390 deaths in 2016 [1]. BC is a major health problem worldwide with approximately 380,000 new cases and estimated 150,000-165000 deaths annually [2].

$\mathrm{BC}$ is a disease of the older population with multiple comorbidities where $90 \%$ of patients are older than 55 at the time of diagnosis [3]. Clinically, BC is categorized as nonmuscle invasive (NMIBC), muscle invasive (MIBC) and metastatic bladder cancers (MBC). NMIBC contributes almost $75-85 \%$ of $\mathrm{BC}$ cases, and $15-25 \%$ of patients have MIBC or metastatic disease at the time of their presentation [4].

Superficial disease is usually treated by rans-urethral resection of the bladder tumor (TURBT) and intra vesical $\mathrm{BCG}$ especially in high risk patients to decrease the risk of recurrence. BCG refractory NMIBC is usually managed by repeat TURBT along with intra vesical chemotherapy or radical cystectomy (RC) in selected cases [5]. MIBC is usually managed by a multi- disciplinary approach via $\mathrm{RC}$ with or without neo-adjuvant/adjuvant chemotherapy. Almost $50 \%$ of MIBC patients will develop metastatic disease despite multi-modality therapy with dismal median survival rates of about 12-14 months after metastatic disease $[4,6]$.

\section{Chemotherapy for Locally Advanced and Metastatic Disease}

Cisplatin based regimens used as first line agents for locally advanced or metastatic $\mathrm{BC}$ result in high response rates but few durable responses with overall survival (OS) from 9-15 months. MBC has limited therapeutic options and poor overall prognosis as 5 years survival rate is only $5 \%$ even with utilization of cisplatin based therapies [7, 8, 9]. Despite survival benefit seen with cisplatin regimens, almost $30-50 \%$ of patients are ineligible for chemotherapy. Chemotherapy is challenging in older patients and those with coexisting medical co-morbidities including renal insufficiency, heart failure, hearing impairment and poor performance status $[8,10]$. Prognosis of patients with disease relapse or progression after Cisplatin based regimen is dismissal with a median survival of about 6 months [11]. Most of second line agents have a response rate (RR) of $20 \%$ or less, and a median progression free survival (PFS) of 2-4 months. Additionally none has demonstrated improvement in OS as the survival rate is approximately 6-9 months with currently used second line agents [9]. Given ineligibility of many patients to receive cisplatin based therapy there has 
been a need to explore more targeted therapies.

\section{PD1/PDL-1 Pathway, a Negative Regulator of $\mathrm{T}$ Cells Responses}

Immune based therapies have revolutionized the management of MBC. Tremendous advances have been made in understanding the PD1/PDL-1 pathway (programmed cell death protein-1 and programmed death ligand-1) and numerous effective therapies have emerged. This review will focus on mechanisms of targeting the PD1/PDL-1 pathway and the new therapies which have emerged over the last few years for this disease.

Cytotoxic CD8 $+\mathrm{T}$ cells recognize tumor specific antigen presented by MHC -1 (major histocompatibility complex) and resultant cytolytic activity eradicates tumor cells (TC). C4+ $\mathrm{T}$ cells exhibit anti-tumor response with cytokine production. Activity of these effector cells is balanced by regulatory Foxp3+ expressing CD4+ T cells (Tregs) [12].

TC escape immune recognition by decreasing MHC-1 expression and overexpressing $\mathrm{T}$ cell check point inhibitory molecules. PD-1 (Programmed cell death protein-1) is inhibitory check point receptor member of immunoglobulin family, primarily expressed on immune cells (IC) and can bind to ligands PD-L1 (Programmed death ligand-1) and PDL2 (Programmed death ligand- 2). PD-L1 is expressed by many normal tissue, TC and IC both in circulation and tumor microenvironment while PD-L2 is mostly expressed by APCs (antigen presenting cells) [13]. PD-L1 binding to PD-1 on lymphocytes inhibits all $\mathrm{T}$ cell responses including activation, proliferation and cytokine production by $\mathrm{T}$ cells. PD-L1 binding to PD-1 on Tregs as abundantly present in many tumors promotes proliferation of these immunosuppressive cells. Blocking the PD-1 pathway decreases immune suppressive activity of Tregs and restores effector $\mathrm{T}$ cell response [36, 42]. PD-L1 expression is inducible on both TC and IC by cytokines especially interferon (IFN) produced by activated $\mathrm{T}$ cells $[12,14]$.

\section{Correlation of PD-L1 Expression with Clinical Response}

Various studies [15-21] have shown increased likelihood of response to PD-1 therapies in PD-L1 positive tumors (those with PD-L1 expression on TC or IC) but fail to identify all responders in these studies as few PD-1 negative tumors also responded to therapy [15-21]. As tumors with low PD-L1 expression are less likely to respond to a single PD-1 blocker, lower or inconsistent expression can identify patients who could get benefit from combination immunotherapy than from a single agent PD-1 inhibitor [17].

In melanoma and renal cell carcinoma (RCC), responses were seen with dual therapy of CTLA-4 (cytotoxic Tlymphocyte associated protein-4) and PD-1 inhibitors regardless of tumor PD-L1 expression which suggests a role of CTL-4 blockers in overcoming resistance to response in
PD-L1 negative tumors [22, 23]. Tumors with high mutation rates are more responsive to PD-1 inhibitors as supported by better response with pembrolizumab in NSCLC with high mutation burden [24]. By exposing neo- antigens, Immunogenic tumors can trigger an immune response with recruitment of $\mathrm{T}$ cells in the tumor micro environment as well as induce high expression of CD8A gene and checkpoint inhibitors like PD-1 and CTLA4 [25]. Tumors with higher numbers of somatic mutations due to mismatch repair defects are more susceptible to check point inhibitors. Pembrolizumab phase 2 showed improved response rate and PFS in mismatch repair deficient colorectal cancer suggesting mismatch repair status as a predictor of response to PD-1 inhibitors [26].

Alteration in the tumor microenvironment and immune system as a result of prior or adjuvant therapies might help in predicting the response to immune inhibitors. In an experimental model, chemotherapy produced local immune suppression via PD-L1 upregulation and better survival was obtained with combination of paclitaxel and a PD1 inhibitor rather than paclitaxel alone. It suggested improved antitumor response with a combination of immunotherapy and chemotherapy [27]. TILs (Tumor infiltrating lymphocytes) PD-L1 expression is strongly associated with response to atezolizumab in solid tumors [15]. The presence of TILs also has been associated with improved outcomes in different tumors like RCC [28] and BC [29, 30] favoring TILs as a potential marker of PD-1 therapy response. Blocking the PD1 pathway itself may not produce anti-tumor effects if the tumor micro environment is lacking those immune cells necessary to eliminate tumor cells despite over-expression of PD-L1 [31].

PD-L1 expression is variable within the same tumor as well as between primary and metastatic lesions suggesting tumor heterogeneity, an important factor of PD-L1 underestimation. Owing to diverse heterogeneity of many tumors, it is reasonable to analyze metastatic lesion PD-L1 status before treatment for response prediction [32].

Careful assessment of tumor characteristics, expression of biologic markers, tumor immune infiltrate, chemokines, neoantigens, mutational analysis, alteration of biologic pathways like mismatch repair may be taken into account in order to predict response to available targeted therapies or immunotherapy [15, 24, 25, 26, 27].

\section{Significance of PD1- PD-L1 Pathway in Bladder Cancer, Perspective and Limitations}

Over the last three decades cisplatin based chemotherapy is the first line treatment and standard of care for MBC. New targeted therapies and biologic agents have not been proven more effective compared to traditional chemotherapeutic agents. Subsequently concept of targeting immune cells developed in order to eliminate tumor tolerance and utilizing the patient's own immune response against tumor antigens 
[33]. Rationale behind the utility of check point inhibitors in $\mathrm{BC}$ is the highly immunogenic behavior of $\mathrm{BC}$ and evidence of successful experience with BCG, utilized to eradicate carcinoma in situ (CIS) and decrease risk of recurrence in NMIBC [34]. Urothelial tumors are dynamic with high rates of somatic and driver mutations and are prone to acquire mutations not only during course of their natural disease process but also in response to treatment [35].

Immune hypothesis suggests the immune system has high likelihood to recognize cancer cells as foreign antigens secondary to high rates of somatic mutations in BC. Though urothelial cancer (UC) can evade immune system recognition by down regulating tumor antigen presentation, manipulating immune check points and subsequently inactivating cytotoxic $\mathrm{T}$ lymphocytes responses [15, 34, 36].

Xylinas et al, in an analysis of 302 BC treated with cystectomy, B7-H3 and PD-1 were over expressed in cancer tissue compared to adjacent normal urothelium (58.6\% vs $6 \%$ and $65 \%$ vs $0 \%$ respectively). Tumor PD-L1 expression predicted increased mortality after cystectomy in patients with organ confined disease. Interestingly increased PD-1 and B7-H3 expression but low PD-L1 expression was noted in patients who received BCG therapy before cystectomy [37]. In an analysis of 75 UC cases by Nakananishi et al, PDL1 over expression on TAICs in high grade tumors was significantly associated with high rates of recurrence and both poor overall and recurrence free survival. After $\mathrm{T}$ classification and stage classification as the first two predictors of poor outcome, tumor associated PD-L1 expression was the third important predictor of prognosis, even more significant than WHO grade [38]. In a study of 280 UC patients by Inman, Increased PD-L1 expression was associated with advanced stage and high grade tumors, though intense expression was observed in CIS [36]. In an analysis of $300 \mathrm{BC}$ patients by Boorjan, tumor PD-L1 and TILs PD-1 expression were associated with stage progression as tumors with high PD-L1 expression and PD-1+ve immune infiltrate were found to have advanced pathologic stage on cystectomy [39]. Analysis of 160 bladder tumors by Bellmunt showed that PD-L1 expression on tumor infiltrating mononuclear (TIMCs) but not on TC was associated with better OS in those BC patients who developed metastasis and were treated with cisplatin based regimen [40].

Sharma et al, Cytotoxic $\mathrm{T}$ cells concentration correlated with better OS in both MIBC and invasive BC suggesting it as one of the prognostic marker [67]. Geraldine et al, Tumor specimens from 155 patients showed abundant expression of PD-L1 and PD-1 in MIBC compared to normal bladder tissue (59.5\% versus $6.7 \%$ and $60.7 \%$ versus $0 \%$ respectively) and proportionately low expression in NMIBC $(22.5 \%$ and $4.2 \%$ respectively). Though no correlation could have been established between mRNA expression of these genes with survival or prognosis [41].

Overall expression of PD-L1 has been correlated with high grade tumors, progressive disease, increased risk of recurrence and poor survival in urothelial bladder cancers $[36,38,39]$ though a few recent studies do not suggest this marker is associated with disease outcome [29, 41]. PD-1 expression on TC and TILs is associated with advanced pathologic stages in UC [36, 38, 39]. PD-L1 expression itself is quite dynamic with evidence of change in expression during the disease course as well as in response to treatment [36]. Discordance in PD-L1 expression was found between metastatic lymph nodes and the primary tumor (cystectomy) specimen of MIBC patients with no previous exposure to chemotherapy. It suggested need of PD-L1 analysis on a sample before treatment initiation rather than tissue from resected specimen for better assessment of the immune microenvironment. In patients with metastases, tumor heterogeneity cannot be captured by single site biopsy and analysis. Genetic sequencing and biomarker expression of metastatic lesions in $\mathrm{BC}$ may provide better prediction of outcome from a subsequent targeted therapy [42].

Utilizing whole genome m RNA expression profiling, MIBC can be divided into luminal, basal and p53 subtypes, similar to molecular subtypes found in breast cancer. Aggressive NAC (neoadjuvant chemotherapy) provide chances for improved survival in basal MIBC secondary to their chemo responsive behavior. Target therapy in addition to traditional chemotherapy can provide better outcomes in luminal type as many luminal MIBC also respond to NAC. P53 - MIBC, subtype tumors expressing active p53 gene signature are resistant to cisplatin based chemotherapies [43].

PD-L1 expression of TAICs, high mutation load and TCGA (The cancer genome atlas) luminal type II are independently associated with improved response and survival. Role of PD-L1 as a predictive/prognostic biomarker in $\mathrm{BC}$ is under investigation as measurement of this marker varies according to assay and techniques utilized as well as secondary to tumor heterogeneity which requires standardization of histochemical techniques and consideration of multiple samples for PD-L1 assay [19].

\section{PDL-1 and BCG Therapy}

Though intra vesical BCG is standard of care after TURB in NMIBC to prevent recurrence, almost $30-45 \%$ of patients fail to respond or relapse within 5 years of treatment. As interferon (IFN) production by infiltrating lymphocytes increases tumor PD-L1 expression, which could be the etiology behind unresponsiveness or relapse of tumor after BCG therapy [44]. BCG after being internalized into urothelial cells produces an immune response through complex mechanism. Presence of an intact immune system including cytokines and immune cells, attachment/ internalization of BCG into tumor cells and presence of live bacteria are factors vital for BCG effectiveness [45]. Increasing tumor PD-L1 expression in correlation with advancing stage suggests PD-L1 as a prognostic predictor of stage progression independent of tumor grade. Inman analyzed 280 patients with high risk BC and suggested that intra-tumoral PD-L1 expression was a potential reason for BCG resistance as abundant expression of PD-L1 (intense staining $>90 \%$ of cells) was detected in the BCG induced 
granuloma of most of (11 out of 16) BCG refractory patients. Though PD-L1 is expressed in almost $40 \%$ of CIS tumors before BCG therapy, CIS cases that failed BCG therapy manifested an approximately 15-20-fold elevation in PD-L1 expression, more abundant within BCG granulomas. This data supports the hypothesis that rising PD-L1 expression could contribute to decreased efficacy of BCG over the time and facilitates CIS to progress into invasive forms, also proposing a therapeutic role of PD-1 inhibitors in earlier stage tumors and NMIBC [36].

PD-L1 expression was analyzed on a cohort of 39 NMIBC patients who underwent at least 2 sessions of TURBT almost 3 months apart and 23 out of 39 received BCG between first and second TURBT. PD-L1 expression in this cohort did not correlate with BCG therapy suggesting over expression of biomarker with disease relapse and dynamic feature of this marker [46].

\section{PD-L1/ PD-1 Inhibitor Therapy in Bladder Cancer}

Immunotherapy in $\mathrm{BC}$ has come a long way through understanding of immune mechanisms which are pivotal in this disease. We will now review all the recent information regarding the role of $\mathrm{PD}-1 / \mathrm{PD}-\mathrm{L} 1$ inhibitor therapy in patients with MBC. Several PD-1/PDL-1 antibodies including Atezolizumab, Pembrolizumab, Avelumab, Durvalumab and Nivolumab have been studied in BC patients with encouraging efficacy.

\subsection{Atezolizumab}

Atezolizumab is an engineered human IgG anti -PD-L1 antibody which targets PD-L1 expressed on TC and IC, blocking interaction of PD-L1 with PD-1. Fc domain in MPDL3280 is modified to avoid antibody dependent cellular toxicity, thus preventing depletion of PD-L1 expressing T cells.

Phase Ib study (67 patients) achieved $43 \%$ response rate in patients with positive PD-L1 status (IHC 2/3) and significant $11 \%$ response in negative/ weak PD-L1 status patients (IHC 0/1) through 6 weeks, 52\% achieved response in (IHC 2/3) group at 12 weeks follow up [16]. Dose expansion phase Ia (92 patients) resulted in ORR of $50 \%$ in IHC $2 / 3$ group and $17 \%$ in IHC $0 / 1$ group. OS at 1 year was $57 \%$ for IHC $2 / 3$ versus $38 \%$ for those in IHC $0 / 1$ category [18]. Phase II study with Atezolizumab was conducted based on better response rate and survival associated with high PD-L1 expression on IC in phase I.

Phase II trial IMVior 210 included a total of 429 patients who were platinum ineligible, previously untreated or belonged to platinum refractory category. Patients were divided into 2 cohorts based on their prior exposure to platinum based therapy.

IMVigor 210 cohort 2 (NCT 02108652) evaluated Atezolizumab (1200 mg Q 3 weeks) in 310 platinum refractory and poor prognostic patients $(78 \%$ with visceral metastasis and $21 \%$ had $\geq 3$ regimens). PD-L1 status was categorized as IC $<1 \%$, IC1 $\geq 1 \%$ but $<5 \%$, IC $2 / 3 \geq 5 \%$ based on percentage of PD-L1 positive IC. Responses were recognized in all groups with ORR (overall response rate) of $16 \%$ in cohort, $28 \%$ in IC2/3 and $19 \%$ in IC $1 / 2 / 3$ respectively. Durable responses were seen in poor prognostic patients and $71 \%$ had ongoing responses but MDOR (median duration of response) was not reached at 17.5 months. 1-year OS rate is $37 \%$ overall, $50 \%$ and $40 \%$ for IC $2 / 3$ and IC $1 / 2 / 3$ respectively. Median OS is 7.9 overall, 11.9 and 9 months for IC $2 / 3$ and $\mathrm{IC} 1 / 2 / 3$ respectively. Better ORR is seen in the absence of visceral $(10 \%$ versus $31 \%)$ and liver metastasis (5\% versus $19 \%$ ) with high complete response (CR) (1\% versus $18 \%$ ) achieved in the absence of visceral metastasis at baseline similar to Phase I trial [58]. Grade 3-4 TRAEs (treatment related adverse events) occurred in 16\% patients and grade 3/4 IRAEs (immune related adverse events) in 5\%. TRAEs required steroids in $22 \%$ and treatment discontinuation in $4 \%$ patients. There was no treatment related death, immune related renal toxicity or grade 5 TRAEs [19].

Increased PD-L1 expression on IC was associated with high response rate and survival. Markers of $\mathrm{T}$ eff activation including CD8 $+\mathrm{T}$ cell infiltration, IFN induced chemokines (CXCL9, CXCL10), positively correlated to PDL1 expression, but also associated with response to atezolizumab. Other immune regulators including Tregs cells, immune check points, baseline IFN gamma induced gene expression, IFN gamma inducible MHC-I antigen processing and transport gene expression are associated with response to atezolizumab. A study suggested TCGA molecular subtype and mutational load as an important predictor of response to atezolizumab [19]. Atezolizumab came out as a potential second line agent for UC who had disease progression on platinum based therapies.

FDA granted approval to atezolizumab for the treatment of patients with locally advanced or metastatic UC who have disease progression on platinum based chemotherapy on May 18th, 2016. However, the phase III IMvigor 211 trial, (NCT02302807) which included 931 patients with previously treated metastatic UC who progressed during or following a platinum based therapy failed to meet its primary end point of OS. Patients were randomized to atezolizumab or investigators choice of chemotherapy. Further details of this trial will be presented later this year.

Phase 2, IMvigor210 cohort 1: (NCT 02108652) Atezolozumab (1200mg Q 3 weeks) in 119 previously untreated cisplatin ineligible patients with UC achieved overall response in $23 \%$ and $\mathrm{CR}$ in $9 \%$. While $70 \%$ responders had ongoing responses, MDOR was not reached in this cohort or any subgroups. Comparable responses were seen in all PD-L1 subgroups $28 \%$ in IC2/3, 24\% in IC1/2/3, $21 \%$ each in IC0 and IC1 subgroups. Median PFS was 2.7 months, better with high PD-L1 status, 4.1 months in IC2/3 versus 2.1-2.6 in IC1 and IC0 respectively. Median OS was 15.9 months and $12-$ month survival was $57 \%$. Similar to IMvigor210 cohort 2 responses though seen in all TCGA 
subtypes are more significant in luminal type II. Higher mutational load in responders and those with long survival suggested mutation load positive correlation with better response and survival. Grade 3-4 TRAEs occurred in 16\% and grade 3-4 IRAEs in 7\% patients. TRAEs led to therapy interruption in $34 \%$ of patients, discontinuation in $8 \%$. One death related to treatment events is due to grade 5 sepsis (1\%). Statistically significant response seen at follow up of 17 months compared to initial analysis at 6 months suggests checkpoint therapy responses might be delayed requiring longer follow up [20]. Better median OS of 15.9 months compared to gemcitabine- carboplatin (9.3 months) [47] or Cisplatin based regimen (around 15.8 months) [48] and durable responses with manageable toxicity proposed it as a potential first line agent for platinum therapy ineligible patients. FDA granted approval to atezolizumab as a frontline therapy for cisplatin-ineligible patients with locally advanced or metastatic UC in April 2017. Phase 3 IMvigor 130 will compare atezolizumab as monotherapy or in combination with platinum based regimen in patients with locally advanced or metastatic urothelial BC (NCT 02807636). Another Phase III study will evaluate efficacy of atezolizumab as an adjuvant therapy compared to observation in PD-L1 positive MIBC at high risk of recurrence after cystectomy NCT 02450331 (IMvigor 010).

\subsection{Pembrolizumab (MK-3475)}

Pembrolizumab is a highly selective humanized IgG4 antibody against PD-1 which inhibits interaction between PD-1 and ligands PD-L1 and PD-L2.

MK-3475 (NCT01848834) Phase Ib trial (27 evaluable patients) resulted in ORR of $26 \%, 11 \%$ with $\mathrm{CR}, 15 \%$ with partial response (PR) and stable disease (SD) in 15\%. [49]. Median OS of 13 months with pembrolizumab was better than median survival of 6-9 months seen with second line single or combination chemotherapy agents [50]. Study suggests that PD-L1 expression of TC and TAICs might be more reliable marker to identify potential responders to PD-1 inhibitor therapy [49].

KEYNOTE-045 Phase 3 trial (NCT 02256436) 542 platinum refractory UC patients were randomly assigned to receive pembrolizumab (200mg Q 3 weeks) or chemotherapy of investigator's choice. Study resulted in a high response rate $(21.1 \%$ vs $11.4 \%$ ), longer Median OS (10.3 vs 7.4 months) and lower rate of TRAEs $(60.9 \%$ vs $90.2 \%)$ with pembrolizumab compared to second line chemotherapy. Grade $\geq 3$ TRAEs rate was $15 \%$ in pembrolizumab that required discontinuation of therapy in $5.6 \%$ versus $49.9 \%$ events in chemotherapy arm that resulted in therapy discontinuation in $11 \%$ patients. MDOR was not reached in pembrolizumab cohort but was 4.3 months in chemotherapy arm. Durable responses were observed with pembrolizumab; $68 \%$ responders estimated to have ongoing responses for $\geq 1$ year in pembrolizumab group versus $35 \%$ expected to maintain responses for $\geq 1$ year in chemotherapy arm. PDL1 combined positive score (CPS) is defined as percentage of PDL1 expressing IC and TC out of total number of TC.
There was no significant difference in PFS between chemotherapy and pembrolizumab groups (3.3 versus 2.1 respectively) or among patients with CPS> $10 \%$. Pembrolizumab showed better outcome over chemotherapy in all subgroups irrespective of PDL-1 expression including patients with liver metastases. [51]. On May 18, 2017 FDA approved pembrolizumab for patients with locally advanced or metastatic urothelial carcinoma who have disease progression during or following platinum-containing chemotherapy.

KEYNOTE 052 (NCT 02335424 phase 2 study) Pembrolizumab (200 mg Q 3 weeks) as a first line agent in patients with advanced/ metastatic UC resulted in ORR of $24 \%$ in first 100 patients, $37 \%$ in patients with high CPS $\geq 10 \%$ and $25.4 \%$ in patients with CPS $\geq 1 \%$. CR is $6 \%$ in all patients, $13.3 \%$ in patients with CPS $>10 \%$ and $6.3 \%$ in CPS $\geq 1 \%$. Grade 3-4 TRAEs experienced by $16 \%$ required discontinuation of treatment in $5 \%$ of patients. MDOR is not reached yet [52].

Few ongoing trials will highlight efficacy of pembrolizumab in both organ confined and metastatic disease. MK-3475-057 / KEYNOTE-057(NCT02625961) phase II trial will determine efficacy of pembrolizumab in high risk NMIBC who are unresponsive to BCG. A Phase II trial will assess ORR of Pembrolizumab as a first line agent in patients with unresectable and metastatic UC ineligible for cisplatin based chemotherapy (KEYNOTE 052; NCT02335424). Another phase 3 study would determine efficacy in term of PFS/ OS for pembrolizumab with or without chemotherapy versus chemotherapy alone in advanced/ metastatic UC (MK-3475-361/KEY-NOTE-361) NCT 02853305. Another Phase I study will evaluate pembrolizumab in combination with $\mathrm{BCG}$ for high risk NMIBC (NCT 02324582).

\subsection{Avelumab}

Avelumab is a human anti-PD-L1 (IgG1) antibody which binds to both human and mouse PD-L1 with high affinity. Avelumab has significantly reduced tumor size and burden with improved long- term survival in mice where MB49 tumor cells were introduced in mice bladder resulting in tumor development. Combination of BCG and avelumab provided no additional benefit in reducing tumor burden over avelumab alone. PD-L1 expression was high in bladder tumor tissue whether or not they were treated with avelumab but low expression was found in bladder tissue and tumor/ bladder junction of mice who responded. Mice with complete tumor resolution after avelumab were protected against tumor re challenge with intravesical MB49 tumor cells re instillation, suggesting avelumab also induces $\mathrm{T}$ cell memory response. Avelumab was less effective in mice depleted of either CD4 or CD8; this suggests need of intact immune system for immune inhibitors response [53]. Avelumab could be a promising therapy for NIMB and CIS especially in those who failed to respond to $\mathrm{BCG}$, but it requires further studies.

Phase Ib study evaluated (MSB0010718C) avelumab $(10 \mathrm{mg} / \mathrm{kg}$ every 2 weeks) in platinum ineligible or refractory 
UC patients with $\geq 2$ prior treatments (NCT01772004). As of March 2015, among 44 evaluable patients, overall response was reported to be $15.9 \%$, one patient with $\mathrm{CR}$ and six with PR. Patients were not selected based on PDL-1 expression and status (positive status defined as $\geq 5 \%$ expression on TC). Disease control rate (CR+SD+PR) was $59.1 \%$ based on $42.3 \%$ with stable disease (SD) and $15.9 \%$ patients with responses $(\mathrm{CR}+\mathrm{PR})$. At the time of data cut off, 6 responders had ongoing response to therapy but MDOR could not be reached. TRAES of any grade occurred in $60 \%$ of patients, noticeable events were Grade 1-2 infusion related reaction in $18.2 \%$, fatigue in $15.9 \%$ and grade 3 asthenia in one patient. Anti-tumor activity including both better ORR (40\% versus $9.1 \%)$ and PFS (70\% versus $45.5 \%)$ was seen in PDL1+ve status patients [54]. On May $9^{\text {th }}, 2017$ FDA granted approval to avelumab for patients with locally advanced or metastatic UC with disease progression during or following platinum based chemotherapy.

Few prospective trials would further explore efficacy of avelumab in UC. A Phase I study will evaluate activity of avelumab in locally advanced/ metastatic solid tumors including UC (NCT 01772004). Another Phase III trial will compare survival with avelumab plus best supportive care versus supportive care in patients who achieved a response or stable disease after completion of first line chemotherapy cisplatin/ carboplatin or gemcitabine regimen (NCT 02603432).

\subsection{Nivolumab}

Nivolumab is an IgG4 immnuoglobulin, after binding to PD-1 receptor inhibits its interaction with ligands PD-L1 and PD-L2.

Phase I/II trial (Check Mate 032) NCT 01928394 Nivolumab $(3 \mathrm{mg} / \mathrm{kg}$ Q 2 weeks) in 78 platinum refractory UC patients, obtained ORR of $24.4 \%$ irrespective of PD-L1 status. Median PFS was 2.8 month with 1- year PFS of $21 \%$. Median OS of 10 months in those with TC PDL-1 expression of $<1 \%$ versus 16 months in those with PDL-1 expression $>1 \%$; needs longer follow up is needed to clarify this difference [55].

Phase 2 trial Check Mate 275 (NCT 02387996) Nivolumab ( $3 \mathrm{mg} / \mathrm{kg}$ IV Q 2 weeks) in 270 patients of locally advanced or metastatic UC obtained ORR of $19.6 \%$ (in 52 out of 265 evaluable patients) irrespective of their PDL1 status, $2 \%$ with CR and $17 \%$ with PR. ORR was $28.4 \%$ in patients with PDL1 expression $\geq 5 \%, 23.8 \%$ in PDL1 expression $\geq 1 \%$ and $16.1 \%$ in PD-L1 expression $<1 \%$. MDOR was not reached with ongoing response in $77 \%$ of responders at time of analysis in June 2016. Median PFS was 2 months. Median OS was 11.3 months in those with PD-L1 expression $\geq 1 \%$ and 5.95 months with PD-L1 expression $<1 \%$. Better response was seen in patients with few Bellmunt risk factors compared to those with more risk scores. Grade 3-4 TRAEs experienced by $18 \%$ of patients and $5 \%$ required discontinuation of therapy. Three deaths $(2.1 \%)$ caused by TRAEs were secondary to cardio-respiratory failure and pneumonitis.
This study identified further potential markers for immune therapy response. Higher expression of several biomarker including CXCL9, CXCL10, CD8 and 12 chemokines signatures were recognized in nivolumab responders. High interferon signature score as found in basal 1 subtype is associated with high likelihood of response to nivolumab as $30 \%$ of responders had basal type 1 [56]. Better OS of 8.74 months was seen with nivolumab versus 6.98 months survival from second line chemotherapy agents [57].

FDA granted approval to nivolumab for treatment of patients with locally advanced or metastatic UC who had disease progression during or following platinum- based chemotherapy, in February 2, 2017.

Phase I/II Check Mate 032 study, Combination of nivolumab with ipilimumab has shown better response rate (RR) of $38.5 \%$, CR $3.8 \%$ in cohort A (higher dose ipilimumab $3 \mathrm{mg} / \mathrm{kg}$ plus nivolumab $1 \mathrm{mg} / \mathrm{kg}$ ) versus RR of $26 \%$, CR $2.9 \%$ in cohort B (lower dose ipilimumab $1 \mathrm{mg} / \mathrm{kg}$ plus nivolumab $3 \mathrm{mg} / \mathrm{kg}$ ). OS is 10.2 months in cohort A versus 7.3 in Cohort B. Grade 3/4 TRAEs rates were similar in each cohort, at $30.8 \%$ and $31.7 \%$, for the cohort $\mathrm{A}$ and cohort $\mathrm{B}$, respectively. Both better response and survival were achieved with higher dose ipilimumab combination with nivolumab [58].

Various ongoing trials would further evaluate efficacy and safety of nivolumab.

A Phase I/II trial is evaluating efficacy of nivolumab alone or in combination with ipilimumab in advanced/ metastatic solid tumors including BC. (NCT01928394). Another Phase 3 (Check Mate 274) NCT 02632409 will evaluate efficacy and safety of nivolumab compared to placebo in patients with invasive BC after radical surgery. A Phase 2 NCT02387996 will determine the effect of nivolumab (BMS-936558) for reducing tumor burden in patients with platinum refractory BC.

\subsection{Durvalumab (MEDI4736)}

Durvalumab is human monoclonal antibody that targets PD-L1 ligand.

Phase I /II heavily pretreated 61 patients of BC (93.4\% with on $\geq 1$ systemic therapy) and $31.1 \%$ ( $\geq 3$ prior therapies) received Durvalumab (10mg/kg Q 2 weeks). PDL1 expression on $>25 \%$ of TC or IC was cut off for positive status in either TC or IC subgroup or in combined TC/IC category. ORR was $31 \%$ in 42 evaluable patients with ORR of $46.4 \%$ in PD-L1 positive and ORR of $0 \%$ in PD-L1 negative group. DCR at 12 weeks was $57.1 \%$ in PDL+ve versus $28.6 \%$ in PDL-ve subgroups. Among PDL1 +ve group ORR is better in those with lymph node disease compared to those with liver metastases (ORR $66.7 \%$ vs 37.5 ). At data cut off Nov 2015, (92.3\%) 12 out of 13 responders had ongoing response though MDOR was not reached. Grade 3 TRAEs in $5 \%$ including infusion reactions, tumor flare and AKI $(1.6 \%$ each) requiring steroids. No grade 4/5 TRAEs were reported. Study suggests either independent TC/IC or combined TC/IC PD-L1 status to be considered for response prediction compared to previous studies stressing IC PD-L1 status for 
response prediction [59].

Durvalumab received FDA approval for locally advanced or metastatic UC with disease progression after platinum based regimens in May 2017 based on Phase I/II study. Durvalumab is being evaluated as a first line agent in Phase 3 study either as a monotherapy or in combination with tremelimumab versus standard chemotherapy for Stage 4 bladder cancer (NCT02516241).

\section{Conclusion}

PD-1 inhibitors have promising results for advanced and refractory bladder tumors which has given new hope for treatment of metastatic cancer as there has not been a significant standard second line therapy for $\mathrm{MBC}$ in past 30 years. Better response correlated with PD-L1 expression and absence of visceral metastasis at baseline in bladder cancer trials but durability of responses still needs to be determined. Current focus should be to identify potential biomarkers which could predict likelihood of response to avoid unnecessary toxicity. Checkpoint inhibitors in combination with other agents targeting VEGF, MET, HER2 and FGF, CD105 and radiation might improve out come in patients with bladder cancer which requires further evaluation.

Table 1. Inhibitors trials in patients with bladder cancer.

\begin{tabular}{|c|c|c|c|c|}
\hline PD1/PDL-1 inhibitor/ Trial & Dose & $\mathbf{N}$ & ORR\% & MTR \\
\hline $\begin{array}{l}\text { Atezolizumab } \\
\text { Phase II IM Vigor } \\
\text { Cohort } 2 \\
\text { NCT } 02108652 \\
{[19]}\end{array}$ & 1200mg IV Q 3 wks & 310 & $\begin{array}{l}16 \% \\
\text { IC } 1 / 2 / 319 \% \\
\text { IC } 2 / 328 \% \\
9.5 \% \text { in those with PDL-1 expression }<5 \\
26 \% \text { in those with PDL-1 expression } \geq 5 \% \\
\text { CR } 7 \%\end{array}$ & $2.1 \mathrm{M}$ \\
\hline $\begin{array}{l}\text { Atezolizumab } \\
\text { Phase II } \\
\text { IM vigor } 210 \\
\text { Cohort } 1 \\
\text { NCT02108652 } \\
{[20]}\end{array}$ & 1200mg IV Q 3 weeks & 119 & $\begin{array}{l}23 \% \\
\text { IC } 2 / 3 \\
28 \% \\
\text { IC } 1 / 2 / 3 \\
24 \% \\
\text { IC } 0 / 121 \% \\
\text { CR } 9 \%\end{array}$ & $2.1 \mathrm{M}$ \\
\hline $\begin{array}{l}\text { Avelumab } \\
\text { Phase Ib } \\
\text { NCT01772004 } \\
{[54]}\end{array}$ & $\begin{array}{l}10 \mathrm{mg} / \mathrm{kg} \\
\mathrm{Q} 2 \text { weeks }\end{array}$ & 44 & $\begin{array}{l}15.9 \% \\
40 \% \text { in PDL- } 1+\text { ve patients } \\
(\geq 5 \% \text { expression TC) } \\
9.1 \% \text { in } \\
\text { PDL- } 1 \text {-ve patients }(<5 \% \text { expression TC) } \\
\text { PR } 6 \text { patients } \\
\text { CR } 1 \text { patient }\end{array}$ & NR \\
\hline $\begin{array}{l}\text { Pembrolizumab } \\
\text { Phase III } \\
\text { Key Note } 045 \\
\text { (Total } 542 \text { patients) } \\
\text { NCT02256436 } \\
\text { [51] }\end{array}$ & 200mg Q 3 weeks & 266 & $21 \%$ & $2.1 \mathrm{M}$ \\
\hline $\begin{array}{l}\text { Durvalumab } \\
\text { Phase I/II } \\
\text { NCT01693562 } \\
{[59]}\end{array}$ & $10 \mathrm{mg} / \mathrm{kg}$ Q 2 weeks & 61 & $\begin{array}{l}31 \% \\
46.4 \% \text { in PDL- } 1+\text { ve patients } \\
\text { (expression } \geq 25 \% \text { TC/IC) } \\
0 \% \text { in PDL- } 1 \text {-ve patients } \\
\text { (expression }<25 \% \mathrm{TC} / \mathrm{IC} \text { ) }\end{array}$ & $\begin{array}{l}6.3 \\
\text { weeks }\end{array}$ \\
\hline $\begin{array}{l}\text { Nivolumab } \\
\text { Phase II } \\
\text { Check Mate } 275 \\
\text { NCT02387996 } \\
\text { [56] }\end{array}$ & $3 \mathrm{mg} / \mathrm{kg}$ IV Q 2 weeks & 265 & $\begin{array}{l}19.6 \% \\
28 \% \text { in patients with PD-L1 expression } \geq 5 \% \\
23 \% \text { in those with PDL-1 } \\
\text { expression } \geq 1 \% \\
16 \% \text { in those with PD-L1 expression }<1 \% \\
\text { CR } 2 \% \\
\text { PR } 17 \%\end{array}$ & $1.87 \mathrm{M}$ \\
\hline
\end{tabular}

Table 1. Continued.

\begin{tabular}{llll}
\hline PD1/PDL-1 inhibitor/ Trial & PFS & OS & AEs \\
\hline Atezolizumab & & Median OS $7.9 \mathrm{M}$ & any grade $70 \%$ \\
Phase II IM Vigor & & $11.9 \mathrm{M}$ for & TRAEs $3 / 4$ \\
Cohort 2 & median PFS 2.1 M & IC $2 / 3$ & $16 \%$ \\
NCT 02108652 & & $9 \mathrm{M}$ for IC1/2/3 & IRAEs $3 / 4$ \\
{$[19]$} & & 1 year- OS $37 \%$ & $6 \%$ \\
Atezolizumab & median PFS 2.7 M & Median OS $15.9 \mathrm{M}$ & any grade $66 \%$ \\
Phase II & $4.1 \mathrm{M} \mathrm{IC2/3}$ & TRAEs Grade $3 / 416 \%$ \\
IM vigor 210 & $2.1 \mathrm{M} \mathrm{IC1}$ & $1-$ year OS $57 \%$ & IRAE Grade $3 / 4$ \\
Cohort 1 & $2.6 \mathrm{M} \mathrm{IC0}$ & & $6 \%$ \\
\hline
\end{tabular}




\begin{tabular}{|c|c|c|c|}
\hline PD1/PDL-1 inhibitor/ Trial & PFS & OS & AEs \\
\hline $\begin{array}{l}\text { NCT02108652 } \\
{[20]}\end{array}$ & & & IRAEs Grade 5 \\
\hline Avelumab & & & \\
\hline Phase Ib & PFS at 12 weeks & & any grade $59 \%$ \\
\hline NCT01772004 & $70 \%$ in PDL-1+ve & NR & Grade 3 TRAEs \\
\hline [54] & $45 \%$ in PDL-1 -ve & & $2 \%$ \\
\hline \multicolumn{4}{|l|}{ Pembrolizumab } \\
\hline Phase III & \multirow{3}{*}{ Median PFS 2.1 M } & \multirow{3}{*}{$\begin{array}{l}\text { Median OS } \\
10.3 \mathrm{M} \\
1 \text { - year OS 43.9\% }\end{array}$} & Any grade $60.9 \%$ \\
\hline Key Note 045 & & & TRAEs Grade $\geq 315 \%$ \\
\hline $\begin{array}{l}\text { (Iotal 542 patients) } \\
\text { NCT02256436 } \\
\text { [51] }\end{array}$ & & & IRAEs grade $3 / 44.5 \%$ \\
\hline Durvalumab & \multirow{4}{*}{ NR } & \multirow{4}{*}{ NR } & TRAEs Any grade $63.9 \%$ \\
\hline Phase I/II & & & TRAEs Grade 3 \\
\hline NCT01693562 & & & $5 \%$ \\
\hline [59] & & & No grade $4 / 5$ event \\
\hline Nivolumab & \multirow{5}{*}{ median PFS 2 M } & Median OS 8.7M & \multirow{5}{*}{$\begin{array}{l}\text { TRAEs any grade } 64 \% \\
\text { Grade } 3 / 4 \text { TRAEs } 18 \%\end{array}$} \\
\hline Phase II & & 11.3 $\mathrm{M}$ those with PDL-1 & \\
\hline Check Mate 275 & & expression $\geq 1 \%$ & \\
\hline NCT02387996 & & $5.9 \mathrm{M}$ those with & \\
\hline [56] & & PD-L1 expression < 1 & \\
\hline
\end{tabular}

$\mathrm{N}=$ number of patients, $\mathrm{MTR}=$ median time to response, $\mathrm{AEs}=$ adverse events

TRAEs $=$ treatment related adverse events, $I R A E=$ immune related adverse events, $O R R=$ overall response rate

$\mathrm{CR}=$ complete response, $\mathrm{PR}=$ Partial response, $\mathrm{OS}=$ overall survival

$\mathrm{PFS}=$ progression free survival, $\mathrm{M}=$ months, $\mathrm{TC}=$ tumor cells, $\mathrm{IC}=$ immune cells, $\mathrm{IV}=$ intravenous

\section{References}

[1] Siegel RL, Miller KD, Jemal A. Cancer statistics, 2016. CA Cancer J Clin. 2016 Jan-Feb; 66(1): 7-30. doi: 10.3322/caac.21332. Epub 2016 Jan 7. PMID: 26742998.

[2] Jemal A, Bray F, Center MM, et al. Global cancer statistics. CA Cancer J Clin 2011; 61; 69-90.

[3] American cancer society. What are the key statistics about bladder cancer?

http://www.cancer.org/cancer/bladdercancer/detailedguide/bla dder-cancer-key-statistics. Accessed March 11, 2014.

[4] Kim WY. The geriatrics and genetics behind bladder cancer. Am Soc Clin Oncol Educ Book. 2014: e192-e195.

[5] Pinsky CM, Camacho FJ, et al. Intravesical administration of bacillus Calmette-Guérin in patients with recurrent superficial carcinoma of the urinary bladder: report of a prospective, randomized trial. Cancer Treat Rep. 1985 Jan; 69(1): 47-53. PMID: 3881177.

[6] Seront E, Machiels JP. Molecular biology and targeted therapies for urothelial carcinoma. Cancer tret. Rev. 41(4), 341-353 (2015).

[7] Von dr Maase H, Sengelov L, et al. Long term survival results of a randomized trial comparing gemcitabine plus cisplatin, with methotrexate, vinblastine, doxorubicin, plus cisplatin in patients with bladder cancer. J Clin Oncol 2005; 23: 4602-08.

[8] De Santis M, Bellmunt J, et al. Randomized phase II/III trial assessing gemcitabine/carboplatin and methotrexate/carboplatin/vinblastine in patients with advanced urothelial cancer who are unfit for cisplatin-based chemotherapy: EORTC study 30986. J Clin Oncol. 2012 Jan 10; 30(2): 191-9. PMID: 22162575.

[9] Sonpavde G, Sternberg CN, Rosenberg JE, et al. Second-line systemic therapy and emerging drugs for metastatic transitional- cell carcinoma of the urothelium. Lancet Oncol 2010; 11: 861-870.

[10] Dash A, Galsky MD, Vickers AJ, et al. Impact of renal impairment on eligibility for adjuvant cisplatin-based chemotherapy in patients with urothelial carcinoma of bladder. Cancer. 2006; 107: 506-513.

[11] Bellmunt J, Fougeray R, Rosenberg JE, et al. Long-term survival results of a randomized phase III trial of vinflunine plus best supportive care versus best supportive care alone in advanced urothelial carcinoma patients after failure of platinum-based chemotherapy. Ann Oncol. 2013 Jun; 24(6): 1466-72. doi: 10.1093/annonc/mdt 007. PMID: 23419284.

[12] Pardoll Dm. The blockade of immune checkpoints in cancer immunotherapy. Nat. Rev. Cancer 12(4), 252-264 (2012).

[13] Sunshine J, taube JM. PD-1/PD-L1 inhibitors. Curr. Opin. Pharmacol. 23 32-38 (2015).

[14] Francisco LM, Sage PT, Sharpe AH. The PD-1 pathway in tolerance and autoimmunity. Immunol Rev 2010; 236: 219-242.

[15] Herbst RS, Soria JC, Kowanetz M, Fine GD, Hamid O, Gordon MS, et al. Predictive correlates of response to antiPD-L1 antibody MPDL3280A in cancer patients. Nature 2014; 515: 56-67.

[16] Powles T, Eder JP, et al. MPDL3280A (anti-PD-L1) treatment leads to clinical activity in metastatic bladder cancer. Nature. 2014 Nov 27; 515(7528): 558-62.

[17] Topalian SL, Hodi FS, Brahmer JR, et al. Safety, activity, and immune correlates of anti-PD-1 antibody in cancer. N Engl J Med. 2012; 366: 2443-54.

[18] Petrylak, D, Powles T, et al. (2015) A phase Ia study of MPDL3280a (anti-PDL1): updated response and survival data in urothelial bladder cancer (UBC). J Clin Oncol 33, 2015 (Suppl.): abstract 4501.2015 ASCO Annual Meeting. 
[19] Loriot Y, Rosenberg JE, Powles TB, et al. Atezolizumab (atezo) in Platinum (plat)- treated locally advanced/ metastatic urothelial carcinoma (mUC): updated OS, safety and biomarkers from the $\mathrm{Ph}$ II IMvigor210 study. Ann Once. 2016; 27(6): 266-295.

[20] Balar AV, Galsky MD, Rosenberg JE, et al. IMvigor210 Study Group. ancet. 2017 Jan 7; 389(10064): 67-76. doi: 10.101Atezolizumab as first-line treatment in cisplatinineligible patients with locally advanced and metastatic urothelial carcinoma: a single-arm, multicentre, phase 2 trial. L 6/S0140-6736(16)32455-2. PMID: 27939400.

[21] Massard C, Gordon MS, Sharma S, et al. Safety and Efficacy of Durvalumab (MEDI4736), an Anti-Programmed Cell Death Ligand-1 Immune Checkpoint Inhibitor, in Patients With Advanced Urothelial Bladder Cancer. J Clin Oncol. 2016 Sep 10; 34(26): 3119-25. doi: 10.1200/JCO.2016.67.9761. PMID: 27269937.

[22] Wolchok JD, Kluger H, et al. Nivolumab plus ipilimumab in advanced melanoma. N Engl J Med. 2013; 369: 122-133.

[23] Hammers H, plimack E, Infante J, et al. Phase I study of nivolumab in combination with ipilimumab in metastatic renal cell carcinoma (mRCC) J Clin Oncol 2014; 32: (5s): abstr 4505.

[24] Rizvi NA, Hellmann MD, Snyder A, et al. Cancer immunology. Mutational landscape determines sensitivity to PD-1 blockade in non-small cell lung cancer. Science. 2015 Apr 3; 348 (6230): 124-128.

[25] Schumacher TN, Schreiber RD (2015) Neoantigen in cancer immunotherapy. Science 348: 69-74.

[26] Le DT, Uram JN, et al. PD-1 Blockade in Tumors with Mismatch-Repair Deficiency. N Engl J Med. 2015 Jun 25; 372(26): 2509-20. doi: 10.1056/NEJMoa1500596. Epub 2015 May 30

[27] Peng J, Hamanishi J, Matsumura N, et al. Chemotherapy Induces Programmed Cell Death-Ligand 1 Overexpression via the Nuclear Factor- $\mathrm{\kappa B}$ to Foster an Immunosuppressive Tumor Microenvironment in Ovarian Cancer. Cancer Res. 2015 Dec 1; 75(23): 5034-45. doi: 10.1158/0008-5472. CAN14-3098. Epub 2015 Nov 16. PMID: 26573793.

[28] Galon J, Costes A, Sanchez-Cabo F, et al. Type, density and location of immune cells within human colorectal tumors predict clinical outcome. Science. 2006; 313: 1960-1964. (PubMed: 17008531).

[29] Sharma P, Shen Y, Wen S, et al. CD8 tumor-infiltrating lymphocytes are predictive of survival in muscle-invasive urothelial carcinoma. Proc Natl Acad Sci U S A. 2007 Mar 6; 104(10): 3967-72.

[30] Faraj SF, Munari E, Guner G, et al. Assessment of tumoral PD-L1 expression and intratumoral CD8 + T cells in urothelial carcinoma. Urology. 2015 Mar; 85(3): 703.e1-6. doi: 10.1016/j.urology.2014.10.020. PMID: 25733301.

[31] Messina JL, Fenstermacher DA, Eschrich S, et al. 12Chemokine gene signature identifies lymph node structure in melanoma; potential for patient selection for immunotherapy? Sci Rep. 2012; 2: 765.

[32] Callea M, Albiges L, Gupta M, et al. Differential Expression of PD-L1 between Primary and Metastatic Sites in Clear-Cell Renal Cell Carcinoma. Cancer Immunol Res. 2015 Oct; 3(10): 1158-64.
[33] Borghaei H, Smith MR, Campbell KS, Immunotherapy of cancer. Eur. J. Pharmacol. 625(1-3), 41-54(2009).

[34] Chen DS, Mellman I, Oncology meets immunology: the cancer-immunity cycle. Immunity $2013 ; 39: 1-10$.

[35] Gerlinger m, Catto JW, Orntoft TF, et al. Intratumor heterogeneity in urologic cancers: From molecular evidence to clinical implications. Eur Urol 67: 729-737, 2015.

[36] Inman BA, Sebo TJ, Frigola X, et al. PD-L1 (B7-H1) expression by urothelial carcinoma of bladder and BCGinduced granulomata: association with localized stage progression. Cancer 2007; 109: 1499-1505.

[37] Xylinas E, Robinson BD, Kluth LA, et al. Association of Tcell coregulatory protein expression with clinical outcome following radical cystectomy for urothelial carcinoma of bladder. Eur J Surg Oncol 2014; 40(1): 121-7.

[38] Nakanishi j, Wada Y, et al. Overexpression of B7-H1 (Pd-L1) significantly associates with tumor grade and postoperative prognosis in human urothelial cancers. Cancer Immunol. Immunother. 56 (8), 1173-1182 (2007).

[39] Boorjian SA, Sheinin Y, et al. T-cell coregulatory molecule expression in urothelial cell carcinoma: clinicopathologic correlations and association with survival. Clin Cancer Res. 2008 Aug 1; 14(15): 4800-8.

[40] Bellmunt J, Mullane SA, et al. Association of PD-L1 expression on tumor-infiltrating mononuclear cells and overall survival in patients with urothelial carcinoma. Ann Oncol. 2015 Apr; 26(4): 812-7.

[41] Geraldine Pignot, Constance LE Goux, et al. mRNA expression levels and prognostic value of PD1/PDL1 and CTLA4 pathways genes in a large series of 155 bladder tumors. 2016 ASCO Annual Meeting. J Clin Oncol 34, 2016 (suppl; abstr 4523).

[42] Mukherji D, Jabbour MN, Saroufim M, et al. Programmed DeathLigand 1 Expression in Muscle-Invasive Bladder Cancer Cystectomy Specimens and Lymph Node Metastasis: A Reliable Treatment Selection Biomarker? Clin Genitourin Cancer. 2016 Apr; 14(2): 183-7. doi: 10.1016/j.clgc.2015.12.002. Epub 2015 Dec 17. PMID: 26775720.

[43] Choi W, Porten S, Kim S, et al. Identification of distinct basal and luminal subtypes of muscle-invasive bladder cancer with different sensitivities to frontline chemotherapy. Cancer Cell. 2014 Feb 10; 25(2): 152-65. doi: 10.1016/j.ccr.2014.01.009. PMID: 24525232.

[44] Siegel R, Ma J, Zou Z, Jemal A. Cancer statistics, 2014. CA 2014; 64: 9-29.

[45] Ludwig AT, Moore JM, et al. Tumor necrosis factor-related apoptosis-inducing ligand: a novel mechanism for Bacillus Calmette-Guérin-induced antitumor activity. Cancer Res. 2004 May 15; 64(10): 3386-90. PMID: 15150089.

[46] Michael E. Hurwitz, Daniel Peter Petrylak, et al. J Clin Oncol 33, 2015 (suppl; abstr e15504) 2015 ASCO Annual Meeting.

[47] De Santis M, Bellmunt J, et al. Randomized phase II/III trial assessing gemcitabine/carboplatin and methotrexate/carboplatin/vinblastine in patients with advanced urothelial cancer who are unfit for cisplatin-based chemotherapy: EORTC study 30986. J Clin Oncol. 2012 Jan 10; 30(2): 191-9. doi: 10.1200/JCO.2011.37.3571. 
[48] Bellmunt J, von der Maase $\mathrm{H}$, et al. Randomized phase III study comparing paclitaxel/cisplatin/gemcitabine and gemcitabine/cisplatin in patients with locally advanced or metastatic urothelial cancer without prior systemic therapy: EORTC Intergroup Study 30987. J Clin Oncol. 2012 Apr 1; 30(10): 1107-13. doi: 10.1200/JCO.2011.38.6979. PMID: 22370319.

[49] Plimack ER, Bellmunt J, Gupta S, et al. Safety and activity of pembrolizumab in patients with locally advanced or metastatic urothelial cancer (KEYNOTE-012): a non-randomised, openlabel, phase 1b study. Lancet Oncol. 2017 Feb; 18(2): 212220. doi: 10.1016/S1470-2045(17)30007-4. PMID: 28081914.

[50] Sonpavde G, Sternberg CN, Rosenberg JE, Hahn NM, Galsky MD, Vogelzang NJ. Second-line systemic therapy and emerging drugs for metastatic transitional-cell carcinoma of the urothelium. Lancet Oncol. 2010 Sep; 11(9): 861-70. doi: 10.1016/S1470-2045(10)70086-3. Review. PMID: 20537950.

[51] Bellmunt J, de Wit R, et al. KEYNOTE-045 Investigators. Pembrolizumab as Second-Line Therapy for Advanced Urothelial Carcinoma. N Engl J Med. 2017 Mar 16; 376(11): 1015-1026. doi: 10.1056/NEJMoa1613683. Epub 2017 Feb 17.

[52] Balar A, Bellmunt J, O' Donnell PH, et al. Pembrolizumab (pembro) as a first line therapy for advanced/ resectable or metastatic urothelial cancer: preliminary results from the phase 2 KEYNOTE-052 study. European society for medical oncology. Copenhagen, Denmark: Proceeding of ESMO; 2016. Abstr LBA32_PR.

[53] Vandeveer AJ, Fallon JK, et al. Systemic Immunotherapy of Non-Muscle Invasive Mouse Bladder Cancer with Avelumab, an Anti-PD-L1 Immune Checkpoint Inhibitor. Cancer Immunol Res. 2016 May; 4(5): 452-62. doi: 10.1158/23266066. CIR-15-0176. Epub 2016 Feb 26. PMID: 26921031.
[54] Apolo AB, Infante JR, Hamid O, et al. Safety, clinical activity, ad PDL1 expression of avelumab (MSB0010718), an anti-PDL1 antibody, in patients with metastatic urothelial carcinoma from the JAVELIN solid tumor phase Ib trial. J Clin Oncol 2016; 34; abstr 367.

[55] Sharma P, Callahan MK, et al. Nivolumab monotherapy in recurrent metastatic urothelial carcinoma (CheckMate 032): a multicentre, open-label, two-stage, multi-arm, phase $1 / 2$ trial. Lancet Oncol. 2016 Nov; 17(11): 1590-1598. doi: 10.1016/S1470-2045(16)30496-X. PMID: 27733243.

[56] Sharma P, Retz M, Siefker-Radtke A, et al. Nivolumab in metastatic urothelial carcinoma after platinum therapy (CheckMate 275): A multicentre, single-arm, phase 2 trial. Lancet Oncol. 2017 Jan 25. pii: S1470-2045(17)30065-7. doi: 10.1016/S1470-2045(17)30065-7. PMID: 28131785.

[57] Raggi D, Miceli R, Sonpavde G, et al. Second-line singleagent versus doublet chemotherapy as salvage therapy for metastatic urothelial cancer: a systematic review and metaanalysis. Ann Oncol. 2016 Jan; 27(1): 49-61. doi: 10.1093/annonc/mdv509. Review.

[58] Sharma P, Callahan MK, Calvo E, et al. Efficacy and safety of nivolumab plus ipilimumab in metastatic urothelial carcinoma: first results from the phase I/II checkmate 032 study. Society for immunotherapy of cancer (SITC) annual meeting. National Harbor, MD, 2016; abstract 449.

[59] Massard C, Gordon MS, et al. Safety and Efficacy of Durvalumab (MEDI4736), an Anti-Programmed Cell Death Ligand-1 Immune Checkpoint Inhibitor, in Patients With Advanced Urothelial Bladder Cancer. J Clin Oncol. 2016 Sep 10; 34(26): 3119-25. doi: 10.1200/JCO.2016.67.9761. 